\title{
LeanGov.BR: Um método enxuto para suporte ao controle e monitoramento da estratégia de organizações públicas.
}

\author{
Alexsandra Monteiro Silva - http://orcid.org/0000-0002- \\ 5141-4807 - alexsandra ms@yahoo.com.br ${ }^{1}$ \\ Ricardo André Cavalcante Souza - http://orcid.org/0000- \\ 0002-2639-3143 - ricardo.souza@ufrpe.br ${ }^{1}$
}

\begin{abstract}
Resumo - Governança é o sistema pelo qual as organizações são dirigidas, monitoradas e controladas. No contexto do setor público, a governança, orientada por instrumentos legais e normativos, se preocupa com aspectos adicionais como dar transparência sobre o uso e administração da coisa pública e com a prestação de contas dos investimentos transformados em bens e serviços para a sociedade. Para orientar o trabalho de governança existem diversos modelos, frameworks e referenciais, porém esses instrumentos geralmente prescrevem o quê deve ser feito, através de uma série de processos e atividades, mas não especificam um método de como fazer. Diante das especificidades do setor público e da carência por abordagens práticas, este trabalho apresenta a especificação e a experimentação de um método de suporte à governança pública brasileira, fundamentado pelo pensamento Lean e pela agilidade. O método proposto, denominado, LeanGov.BR limita-se a atender ao mecanismo de estratégia da governança, mais especificamente atuar na avaliação, direcionamento e monitoramento do desempenho e resultados organizacionais.
\end{abstract}

Palavras-chaves: Governança, Setor Público, Pensamento Lean, Agilidade.

\section{LeanGov.BR: A Lean method to support strategy controlling and monitoring from public organizations.}

\begin{abstract}
Governance is the system by which organizations are managed, monitored and controlled. In the context of the public sector, governance, guided by legal and normative instruments, is concerned with aspects such as transparency on the use and administration of public things and accountability of investments transformed into goods and services for society. To guide governance work there are models, frameworks and references, but these instruments often prescribe what needs to be done through a series of processes and activities, but they do not specify a method of how to do it. In front of specificities of the public sector and the lack of practical approaches, this work presents the specification and experimentation of a method to support governance in the Brazilian public sector, through lean thinking and agility. The proposed method, called LeanGov.BR, is limited to meeting the governance strategy mechanism, more specifically acting in the evaluation, driving and monitoring of organizational performance and results.
\end{abstract}

Keywords: Governance, Public Sector, Lean Thinking, Agile.

\author{
Data da Submissão: $14 / 12 / 2020$
}

Data de aceitação: 21/04/2021

DOI: 10.51359/2317-0115.2021.249076 


\section{Introdução}

O conceito de Governança está relacionado com mecanismos e responsabilidades através dos quais a autoridade é exercida, decisões são tomadas e a estratégia é coordenada e conduzida nas organizações, sejam elas um país, uma empresa, um setor específico ou um projeto (Luna et al., 2014). A Governança fornece a estrutura por meio da qual os objetivos da empresa são definidos, e os meios para atingir esses objetivos e monitorar o desempenho são determinados (OECD, 2015).

O Tribunal de Contas da União (TCU, 1983) é a instituição brasileira responsável por exercer a fiscalização contábil, financeira, orçamentária, operacional e patrimonial dos órgãos da administração pública. O TCU também prescreve boas práticas e direciona ações para o aprimoramento da governança na administração pública brasileira. Segundo o TCU (2014), Governança Pública compreende essencialmente os mecanismos de liderança, estratégia e controle postos em prática para avaliar, direcionar e monitorar a atuação da gestão, com vistas à condução de políticas públicas e à prestação de serviços de interesse da sociedade. O mecanismo de liderança refere-se a pessoas íntegras, capacitadas, responsáveis e motivadas ocupando os principais cargos das organizações e liderando os processos de trabalho. O mecanismo de estratégia refere-se a escuta ativa das partes interessadas; avaliação do ambiente interno e externo da organização; avaliação e prospecção de cenários; definição e alcance da estratégia; definição e monitoramento de objetivos de curto, médio e longo prazo; alinhamento de estratégias e operações das unidades de negócio e organizações envolvidas ou afetadas. O mecanismo de controle refere-se à avaliação e tratamentos dos riscos organizacionais; e prestação de contas e responsabilização pelos atos praticados.

Para diagnosticar o nível de maturidade em governança das instituições públicas brasileiras, o TCU realiza levantamentos periódicos através da aplicação de questionários nos quais são avaliados aspectos relacionados aos mecanismos de liderança, estratégia e controle. De acordo com o levantamento do TCU (2017) sobre governança pública, 41\% das organizações públicas brasileiras estão em estágio de capacidade inicial em governança pública, mais especificamente em relação aos mecanismos: $38 \%$ estão em estágio de capacidade inicial no mecanismo de liderança, $64 \%$ em estágio de capacidade inicial no mecanismo de estratégia e $35 \%$ em estágio de capacidade inicial no mecanismo de controle. Este diagnóstico mostra que a principal deficiência das organizações públicas brasileiras está no mecanismo de Estratégia Organizacional. Segundo o TCU (2017), esse resultado reflete o baixo nível de capacidade da maioria das instituições públicas em executar um processo de planejamento estratégico, em promover a participação social na governança da organização, em desdobrar o planejamento estratégico entre todas as áreas da organização, e em monitorar e avaliar o desempenho organizacional.

Algumas das deficiências encontradas no mecanismo de Estratégia da Governança Pública podem ser em teoria ser mitigadas com a adoção de princípios e práticas ágeis (Manifesto Ágil, 2001) e do pensamento enxuto (Lean Enterprise Institute, 1998), tais como: planejar para um escopo reduzido, com entregas de valor em menor espaço de tempo; fazer mais com os mesmos recursos; envolver os interessados durante todo o processo; e monitorar e avaliar continuamente o progresso através da entrega de valor e do feedback dos clientes. O pensamento enxuto e a agilidade são paradigmas aplicados com sucesso em áreas como o desenvolvimento de software (Cockburn, 2000) e a Governança de TI (TCU, 2014). 
Neste sentido, o desenvolvimento deste trabalho foi orientado pelo seguinte problema: como reduzir as deficiências encontradas no mecanismo de estratégia da governança de organizações do setor público brasileiro? De modo a tratar esse problema, este trabalho tem então como objetivo geral a especificação e aplicação de um método prático denominado LeanGov. $\mathrm{BR}$, baseado em princípios e práticas ágeis e Lean, visando elevar o nível do controle e monitoramento da estratégia de organizações públicas brasileiras. Para tanto, se fez necessário alcançar os seguintes objetivos específicos: especificar os elementos estruturais do método LeanGov.BR; especificar o workflow do método LeanGov.BR através dos relacionamentos entre os elementos estruturais; desenvolver suporte tecnológico para o método LeanGov.BR e aplicá-lo no contexto de uma organização pública brasileira.

De modo a alcançar o objetivo geral por meio dos objetivos específicos, foram executadas as atividades de um Método de Pesquisa conforme apresentado na Figura 1 em notação do padrão Business Process Model and Notation (BPMN).

Figura 1: Método de Pesquisa do Trabalho

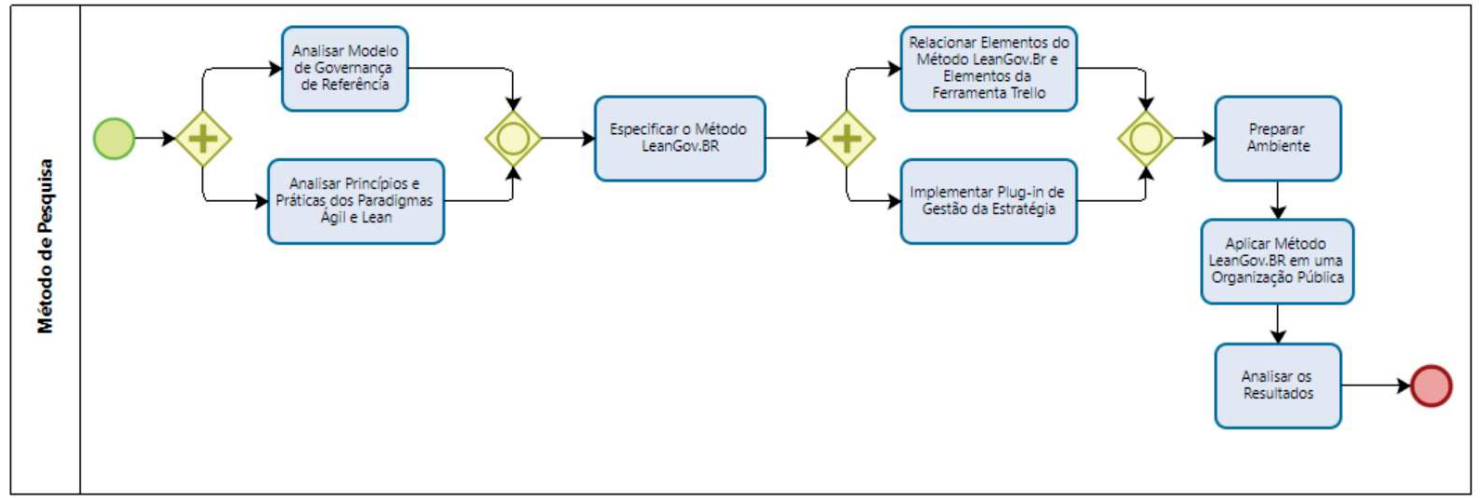

Fonte: Autor (2020)

O método de pesquisa iniciou com a realização das atividades Analisar Modelo de Governança de Referência, para tanto foi utilizado o guia de governança para entidades da administração pública do TCU (2014), e Analisar Princípios e Práticas dos Paradigmas Ágil e Lean, a partir das abordagens Lean Thinking (Shingo, 2007), Manifesto Ágil (2001) e SCRUM (Schwaber, 1995). Ambas as atividades estão consolidadas na Seção 2.

Em seguida na Seção 3 estão sintetizadas as atividades: Especificar o Método LeanGov. $B R$, por meio da definição dos elementos estruturais e comportamentais do modelo por meio de workflow baseado no fluxo do SCRUM; Relacionar Elementos do Modelo LeanGov.BR e da Ferramenta Trello, para descrever como os elementos do modelo podem ser implementados por meio dos elementos da ferramenta de software Trello utilizada para gestão ágil de atividades; Implementar Plug-in de Gestão da Estratégia, para apresentação de painéis gráficos (dashboards) de gestão a partir da leitura do repositório da Ferramenta Trello.

Posteriormente, na Seção 4 são apresentadas as atividades Preparar Ambiente e Aplicar Método LeanGov.BR em uma Organização Pública. A primeira atividade refere-se ao diagnóstico da organização pública quanto aos problemas relacionados ao controle e monitoramento da sua estratégia. Já a segunda, refere-se ao acompanhamento da execução de ciclos de planejamento e execução orientados pelo Método LeanGov.Br com o suporte da ferramenta de software Trello. 
Finalmente, na Seção 5 é apresentada a última atividade desenvolvida do método de pesquisa denominada Analisar Resultados, com as discussões sobre a contribuição do método LeanGov.BR em tratar e reduzir os problemas de controle e monitoramento da estratégia da organização pública estudada.

A Seção 6 do artigo apresenta a Conclusão com as contribuições alcançadas e com as limitações e oportunidades de trabalhos futuros. 2.

\section{Arcabouço Teórico}

O TCU é o órgão do governo brasileiro responsável pela disseminação de boas práticas de gestão e governança pública. O TCU publicou a Estrutura Básica de Governança (TCU, 2014), que visa "reunir e organizar boas práticas de governança pública que, se observadas, podem aumentar o desempenho de entidades públicas". A estrutura de governança do TCU é baseada em boas práticas e modelos internacionais de governança pública, como Boa governança no setor público (IFAC, 2013); Padrão de boa governança para serviços públicos (CIPFA, 2004); COSO (2004; 2013), etc.

A Figura 2 mostra a composição da estrutura do modelo de governança pública do TCU em termos de mecanismos de governança e seus componentes. O mecanismo de Liderança está preocupado com a estrutura de governança da organização e agrupa os seguintes componentes: pessoas e competências; princípios e comportamentos; liderança organizacional; e sistemas de governança. O mecanismo da Estratégia se preocupa em dirigir e monitorar as ações de governança estratégica e é composto de relacionamentos com as partes interessadas; estratégia organizacional; e alinhamento transorganizacional. O mecanismo de Controle está preocupado com a responsabilidade das ações de governança e inclui os seguintes componentes: gerenciamento de riscos e controle interno; auditoria interna; responsabilidade e transparência.

Um conjunto de práticas está vinculado a cada componente da estrutura de governança pública do TCU, a fim de contribuir para a obtenção dos resultados esperados pelas partes interessadas. Alinhado à estrutura de governança do TCU, este trabalho está fortemente associado à prática de "Monitorar e avaliar a execução da estratégia, os principais indicadores e o desempenho da organização" vinculados ao componente Estratégia Organizacional do mecanismo de governança da Estratégia. Essa prática pressupõe que a estratégia da organização e as diretrizes para monitorar e avaliar a implementação dessa estratégia sejam definidas. Além disso, essa prática implica monitorar a implementação de iniciativas estratégicas e avaliar o desempenho da organização, adotando ações de melhoria sempre que necessário. Como resultado da prática, espera-se que a estratégia da organização seja executada de acordo com as metas e objetivos definidos. 
Figura 2 - Modelo de Governança do TCU

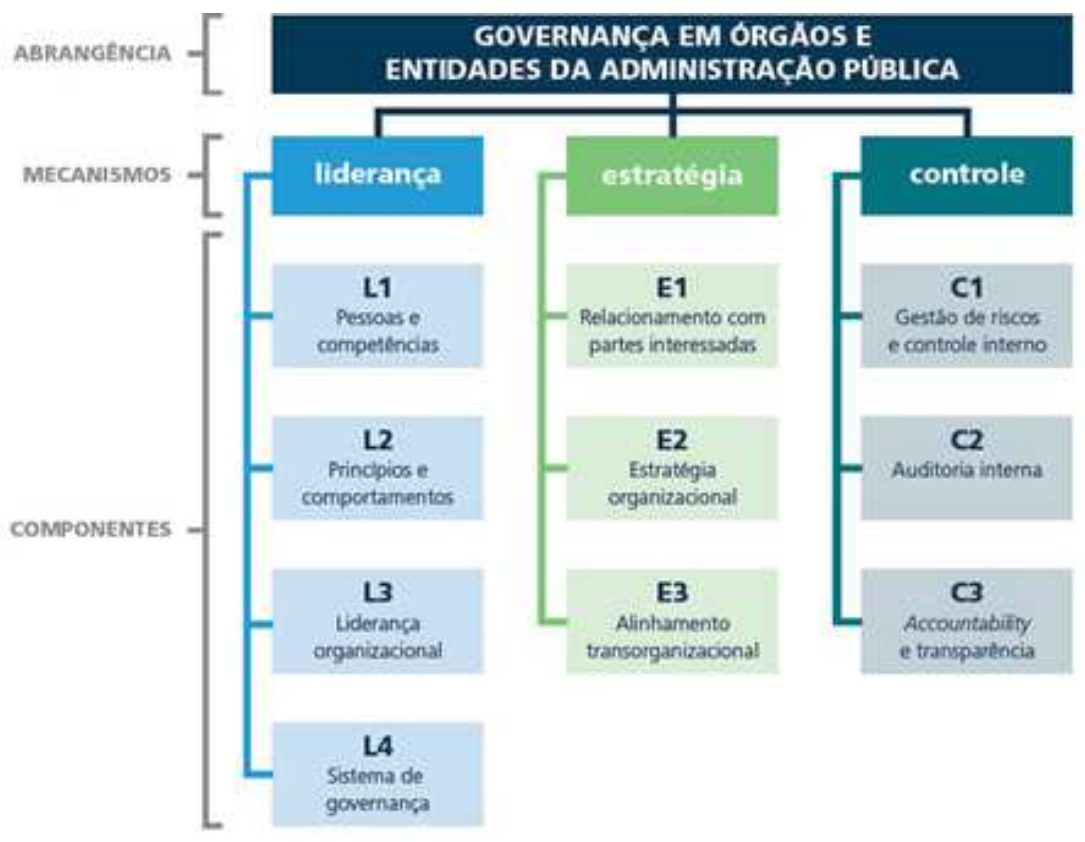

Fonte: TCU (2014).

A estratégia de uma organização é geralmente definida por meio de planejamento estratégico. Planejamento Estratégico é o processo de formulação de estratégias organizacionais, no qual a organização e sua missão são buscadas no ambiente em que está operando (Chiavenato \& Sapiro, 2003). O Planejamento Estratégico é um esforço disciplinado que produz decisões e ações fundamentais que moldam e orientam o que é uma organização, quem ela serve, o que faz e por que faz, com foco no futuro. O Planejamento Estratégico articula não apenas para onde a organização está indo e as ações necessárias para progredir, mas também como saberá se é bem-sucedida (BSI, 1997). O produto do trabalho do Planejamento Estratégico é o Plano Estratégico que consiste em um documento usado para comunicar à organização seus objetivos estratégicos, as metas e ações necessárias para atingir esses objetivos e todos os outros elementos críticos desenvolvidos durante o exercício de planejamento estratégico (BSI, 1997).

As metas e ações são elementos táticos e operacionais, respectivamente, utilizados para orientar e direcionar os esforços das unidades da organização de acordo com a estratégia. Tais elementos do Plano Estratégico podem derivar programas e projetos. Segundo o PMBOK (PMI, 2013), o programa é um grupo de projetos relacionados, gerenciados de maneira coordenada por benefícios e controle estratégicos que não estariam disponíveis se fossem gerenciados individualmente. Projeto é um esforço temporário necessário com a intenção de criar um produto, serviço ou resultado único, com início e propósito definidos, caracterizando sua natureza temporária.

O gerenciamento de projetos consiste no conjunto de atividades, ferramentas e processos utilizados para conduzir os projetos (PMI, 2013). Os paradigmas do gerenciamento de projetos são tradicionais e ágeis. Abordagens tradicionais de gerenciamento de projetos, como PMBOK (PMI, 2013) e PRINCE2 (OGC, 2009) é preditivo e se concentra em um planejamento avançado de todo o escopo do projeto. Abordagens ágeis de gerenciamento de projetos, como OKR (Wodtke, 2016) e SCRUM (Schwaber, 1995), desconsideram a abordagem antecipatória de abordar todo o escopo do projeto e procuram projetar e executar, de maneira iterativa e incre- 
mental, um projeto reduzido escopo de cada vez, em menos tempo e, assim, oferecer resultados mais rápidos.

O paradigma ágil também é aplicado com sucesso no processo de desenvolvimento de software, por exemplo, nas abordagens XP (Beck, 2004) e Crystal (Cockburn, 2000), bem como na Governança de TI, como na abordagem MAnGve (Luna, 2009). O paradigma ágil é baseado e, ao mesmo tempo, complementa outro paradigma chamado Lean, ou Lean Thinking. Segundo Putnik \& Putnik (2012), a agilidade constitui um elemento de apoio ao pensamento enxuto. Lean Thinking é uma filosofia de gerenciamento de manufatura que surgiu no Japão em meados da década de 1960 e ficou conhecida mundialmente por apoiar o Sistema Toyota de Produção (Shingo, 2007). Já o movimento ágil ganhou impulso nos anos 2000 por meio do Agile Manifesto (2001), que visava responder à crise do software (The Standish Group, 1995) por meio de uma maneira ágil de desenvolver software em oposição ao modo prescritivo focado na documentação.

O paradigma Ágil (Agile) é baseado em princípios e práticas. Entre os princípios ágeis estão à satisfação do cliente através da entrega de valor; agregar valor em uma escala reduzida de tempo; construção de projetos em torno de indivíduos motivados; medir o progresso do projeto através da entrega de valor; desenvolver o projeto de forma sustentável; simplificar o trabalho; e melhorar continuamente o desempenho da equipe. Já entre as práticas ágeis estão as iterações de tamanho fixo (timeboxing); reuniões curtas e periódicas para monitorar o progresso do projeto; equipes pequenas, multifuncionais e autogerenciadas; ritmo de trabalho sustentável; e reunião retrospectiva para melhoria contínua.

O Paradigma Lean é independente de tecnologia e busca oferecer vantagens competitivas para a organização, otimizando processos produtivos e incentivando as pessoas a melhorar seus próprios processos de trabalho (Pedrão, 2014). Este paradigma também é baseado em princípios e práticas. Entre os princípios estão à identificação do valor para o cliente, saber no que ele pretende investir; mapear e criar o fluxo de valor para definir um processo lean focado em gerar valor para o cliente; a produção orientada pelas necessidades do cliente, na qual os pedidos orientam o processo de produção e o estoque é praticamente inexistente; e buscar a perfeição, para inserir controles que melhoram a qualidade do fluxo de produção. Já entre as práticas enxutas, há produção na quantidade necessária para suprir a demanda (just-in-time); alinhamento da produção à demanda do mercado (tempo takt); planejamento hoshin (hoshin kanri); remoção de impedimentos (gemba) e controle visual do processo (kanban).

Governança e gerenciamento guiados por paradigmas ágeis e enxutos podem ser auxiliados por meio de ferramentas genéricas de software, como Trello (Trello, 2011). O Trello fornece elementos flexíveis, como tabelas, listas, cartões e listas de verificação, que podem ser adaptados para apoiar o planejamento e o monitoramento da estratégia da organização.

O LeanGov.BR, descrito neste artigo, é um método enxuto que pode servir como instrumento de apoio à governança pública brasileira, mais especificamente, atuando no controle e monitoramento da estratégia organizacional. Para esse fim, esse método utiliza princípios e práticas ágeis e enxutos para o gerenciamento de programas e projetos estratégicos com a assistência tecnológica da ferramenta de software Trello. 


\section{O Método LeanGov.BR}

Método é o uso de procedimentos ou meios para realizar algo, seguindo um planejamento (Michaelis, 2018). A abordagem desenvolvida neste trabalho consiste em um método, chamado LeanGov.BR, para apoiar a governança no setor público brasileiro. Esse método é denominado por basear-se no pensamento enxuto e por estar em conformidade com os instrumentos legais e normativos para as organizações públicas brasileiras.

O método LeanGov.BR é estruturado através de ciclos de planejamento e execução de programas e projetos estratégicos, em alinhamento aos objetivos estratégicos da organização pública, geralmente definidos em instrumentos como o Plano Estratégico Institucional (PEI) ou Plano de Desenvolvimento Institucional (PDI). Um programa agrupa projetos estratégicos e está associado diretamente a um objetivo estratégico. Já um projeto estratégico consiste num desdobramento da estratégia organizacional e contribuem para o alcance das metas de um objetivo estratégico.

O ciclo de planejamento dos programas é orientado pela estratégia organizacional (PEI, PDI, etc.) e consiste na definição e priorização dos programas a serem trabalhados em um ano-base. Para cada programa é definido o Dono de Programa, responsável por promover o alinhamento entre os projetos estratégicos vinculados ao programa. Em seguida, é realizado o ciclo de planejamento dos projetos estratégicos com periodicidade anual devido: a necessidade de prestação de contas oficiais anuais, atender a levantamentos anuais de órgãos de controle, e ao planejamento orçamentário definido pela Lei Orçamentária Anual - LOA (Constituição Federal do Brasil, 1988). Para cada projeto estratégico é designado um Gerente de Projeto Estratégico, responsável por orientar o time e remover os impedimentos e obstáculos que afetam a execução do projeto.

O ciclo de execução dos programas ocorre ao longo do ano através da execução dos respectivos projetos estratégicos associados. $\mathrm{O}$ ciclo de execução de projetos estratégicos baseia-se na abordagem ágil através de iterações mais curtas que visam o alcance de marcos que representam objetivos parciais, específicos e mensuráveis do projeto.

Os ciclos de planejamento e execução de programas e projetos estratégicos do método LeanGov.BR são executados com o auxílio de algumas práticas do pensamento enxuto (lean), tais como: hoshin kanri, para para desdobramento dos objetivos estratégicos e metas em programas e projetos estratégicos, respectivamente; just-in-time, para planejamento e execução de escopo reduzido apenas para a iteração; gemba, para remoção de obstáculos e impedimentos que afetam o trabalho do time, pela liderança; e kanban board (quadro), para gestão à vista por meio da visualização gráfica do progresso de projetos e programas. Na próxima seção é apresentado o fluxo de trabalho (workflow) do ciclo de execução dos projetos estratégicos.

Os elementos estruturais do Método LeanGov.BR referem-se a elementos de engenharia de processo (OMG, 2008) como Ciclos, Fases, Marcos (Milestone), Entregáveis, Tarefas, Papéis, Produtos de Trabalho, Práticas e Ferramentas. 


\subsection{Workflow do método LeanGov.BR}

O workflow do método LeanGov.BR (Figura 3), baseado no fluxo do processo ágil de gestão de projetos SCRUM, descreve a dinâmica iterativa e incremental de execução dos projetos estratégicos. Uma iteração agrupa um conjunto de atividades relacionadas que são repetidas mais de uma vez e serve para organizar o trabalho em ciclos repetitivos (OMG, 2008). Uma iteração inicia com o planejamento da iteração e encerra com a entrega de um incremento de valor ao projeto.

Figura 3 - Workflow do Método LeanGov.BR

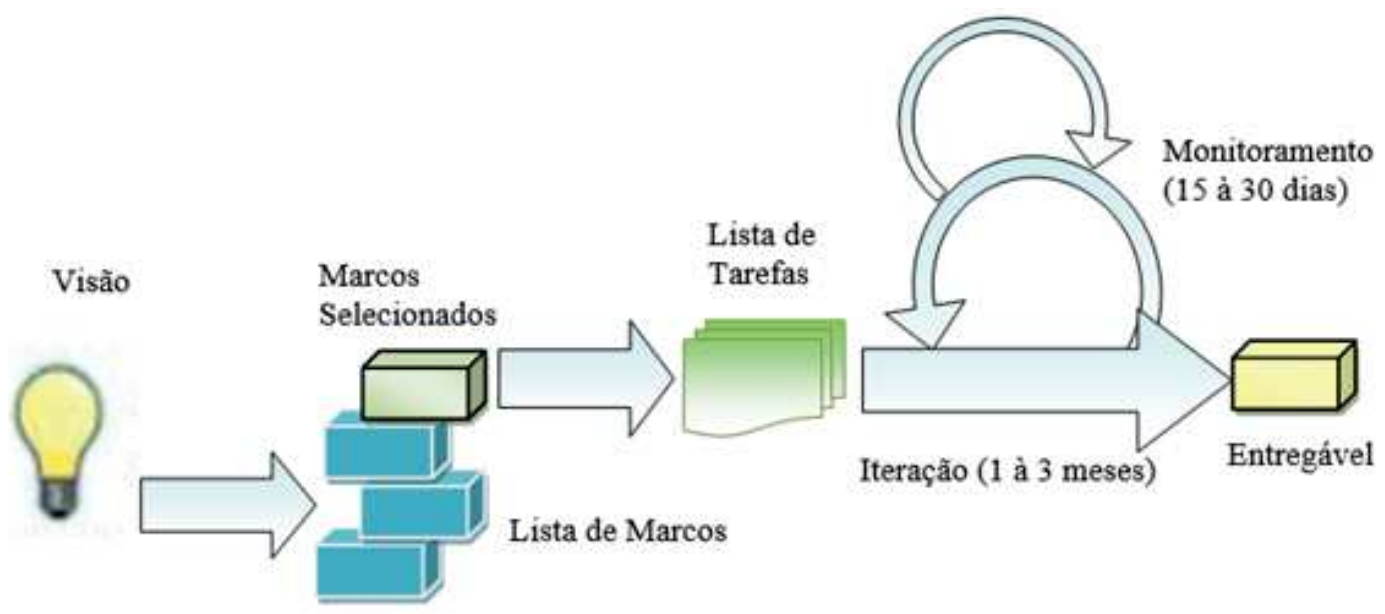

Fonte: Adaptação do Workflow do SCRUM (2017)

O workflow do LeanGov.BR inicia com a elaboração ou atualização da Visão do projeto estratégico pelo Gerente do Projeto Estratégico com o auxílio do Dono do Programa. O resultado consiste em um documento que contém informações gerais do projeto estratégico, tais como, objetivo, escopo, Retorno do Investimento (ROI), alinhamento estratégico, recursos necessários, etc.

Em seguida, é realizada uma reunião de planejamento para definição, atualização e priorização dos marcos do projeto estratégico e principais riscos associados. Esta reunião é mediada pelo Gerente do Projeto Estratégico e participam os membros do time, bem como eventualmente o Dono do Programa, para esclarecer possíveis dúvidas. Esta reunião é dividida em duas partes: a primeira parte para definição do conjunto de marcos do projeto e a segunda para seleção do(s) marco(s) a ser(em) alcançado(s) na iteração.

O resultado da primeira parte da reunião de planejamento é uma Lista de Marcos ordenada por prioridade e uma Lista de Riscos. Um marco (milestone) representa o alcance de um objetivo parcial e serve para medir o progresso de um projeto. Um risco é um evento que se ocorrer, pode causar um impacto positivo ou negativo para o projeto e, portanto, precisa ser monitorado. A Lista de Marcos corresponde ao backlog de marcos que precisam ser alcançados durante o projeto. A Lista de Riscos contém os principais pontos de atenção para o projeto estratégico.

O resultado da segunda parte da reunião de planejamento são os Marcos Selecionados pelo time para serem alcançados na iteração, considerando prioridade, esforço necessário e 
prazo da iteração. Os Marcos Selecionados consistem de um subconjunto da Lista de Marcos e pode conter um ou mais marcos do projeto estratégico. Nesta reunião são ainda discutidas e definidas as restrições do(s) marco(s) selecionado(s), como escopo, custo, etc.

O workflow do método LeanGov.BR prossegue com a realização de uma reunião entre os membros do time, para discussão e definição das tarefas (operacionais) necessárias para o alcance do(s) marco(s) selecionado(s). Uma tarefa representa uma unidade de trabalho assinalável a um responsável, executada com auxílio de práticas e ferramentas. O resultado é a elaboração de uma Lista de Tarefas que contém o conjunto de tarefas a serem realizadas pelo time visando o alcance do(s) marco(s) selecionado(s) para a iteração. O planejamento da iteração encerra com a estruturação de um quadro Kanban com cartões representando as tarefas associadas ao(s) marco(s) para a iteração.

A execução da Iteração consiste na realização das tarefas pelo time em um prazo fixo e não prorrogável (time in box). O prazo da iteração é definido pela organização e pode ser de um mês para projetos simples ou que exigem acompanhamento mais frequente, ou três meses para projetos mais complexos. Durante a Iteração, o quadro Kanban é atualizado à medida que as tarefas mudam de status (ex: pendente, em execução, concluída). Além disso, uma Lista de Conhecimento é continuamente atualizada, contendo as lições aprendidas e boas práticas identificadas ao longo do projeto.

Durante a Iteração, é realizado o Monitoramento periódico do projeto estratégico. Para tanto, são realizadas reuniões a cada quinze dias para os projetos estratégicos considerados mais prioritários ou que o prazo da iteração seja de um mês, ou a cada trinta dias. Nesta reunião os membros do time relatam o progresso e possíveis impedimentos ou obstáculos para a realização das tarefas. O Gerente do Projeto Estratégico media a reunião e pode ser acompanhada pelo Escritório de Projetos, caso a organização adote esta estrutura de apoio aos projetos.

Ao final da iteração é produzido um Entregável, o qual materializa o marco alcançado. A quantidade de entregáveis ao final da iteração está relacionada à quantidade de marcos previstos para serem alcançados. Uma reunião de homologação do entregável é então realizada, na qual os membros do time apresentam os resultados obtidos e o Dono do Programa aceita ou não o entregável. A iteração encerra formalmente com a realização de uma reunião de retrospectiva entre os membros do time e o Gerente do Projeto Estratégico com o objetivo de identificar os pontos positivos e os que precisam ser melhorados para as iterações seguintes. Na próxima seção é apresentado o suporte tecnológico ao workflow do método LeanGov.BR.

\subsection{Suporte Tecnológico ao Método LeanGov.BR}

Os componentes tecnológicos que dão suporte ao workflow do método LeanGov.BR compreendem o meio pelo qual o planejamento é registrado e os programas e projetos estratégicos são monitorados pelos gestores institucionais e pelas equipes técnicas. Esses componentes consistem nas seguintes ferramentas de software: (1) Trello, um aplicativo comercial que fornece estruturas e cartões genéricos que podem ser usados para controlar os marcos e tarefas associadas, a um projeto estratégico; e (2) Numclick, um aplicativo desenvolvido para acompanhar o desempenho do portfólio de projetos vinculados a um programa. 
O Trello (2011) é uma ferramenta colaborativa usada para organizar projetos em quadros que servem para descrever o que está sendo trabalhado, quem está trabalhando no que e onde algo está em processo. A Tabela 1 mostra como os elementos do método LeanGov.BR são representados por componentes da ferramenta de software Trello.

Tabela 1 - Relação entre os elementos do LeanGov.BR e os componentes do Trello

\begin{tabular}{|c|c|c|}
\hline $\begin{array}{l}\text { Elemento } \\
\text { LeanGov.BR }\end{array}$ & $\begin{array}{l}\text { Componente } \\
\text { Trello }\end{array}$ & Descrição \\
\hline $\begin{array}{l}\text { Projeto } \\
\text { Estratégico }\end{array}$ & Quadro & $\begin{array}{l}\text { - Para cada projeto estratégico é criado um quadro no Trello. } \\
\text { - O quadro é nomeado através da seguinte nomenclatura: <sigla do progra- } \\
\text { ma }>\text {.<sequencial }>\text { <nome do projeto }>\text {, por exemplo, PE05_2017.01_Projeto } \\
\text { de Gestão Alimentar. }\end{array}$ \\
\hline $\begin{array}{l}\text { Quadro } \\
\text { Kanban }\end{array}$ & Lista & $\begin{array}{l}\text { - Para cada projeto estratégico é criado um Quadro Kanban } \\
\text { - No quadro referente ao projeto estratégico, são adicionadas três listas com } \\
\text { as seguintes denominações: Planejado, Em execução, Concluído, Cancelado } \\
\text { e Suspenso }\end{array}$ \\
\hline Marco & Cartão & $\begin{array}{l}\text { - Para cada marco da Lista de Marcos do projeto estratégico é criado um cartão } \\
\text { no Trello. } \\
\text { - No quadro referente ao projeto estratégico, são adicionados cartões corres- } \\
\text { pondentes aos marcos. } \\
\text { - No início do projeto, todos os cartões são adicionados à lista Planejado. À } \\
\text { medida que o projeto progride, dependendo do status dos marcos, os cartões } \\
\text { correspondentes são deslocados para as listas Em execução ou Concluído. } \\
\text { - Em cada cartão que representa um marco são adicionados os componentes: } \\
\text { Membros para representar o(s) responsável (is); Data de Entrega para repre- } \\
\text { sentar a data prevista de alcance do marco; e Etiqueta para sinalizar através de } \\
\text { cores o status do marco de acordo com a data de entrega (cinza - no prazo; } \\
\text { amarelo - próxima data de entrega; vermelho - atraso; e verde - concluído). }\end{array}$ \\
\hline $\begin{array}{l}\text { Lista de } \\
\text { Tarefas }\end{array}$ & Checklist & $\begin{array}{l}\text { - A Lista de Tarefas do projeto estratégico é representada através de checklist } \\
\text { no Trello. } \\
\text { - Em cada cartão que representa um marco é adicionado uma checklist reno- } \\
\text { meada como tarefas, a qual irá agrupar os itens que representam as tarefas } \\
\text { relacionadas ao marco. }\end{array}$ \\
\hline Tarefa & $\begin{array}{l}\text { Item da Che- } \\
\text { cklist }\end{array}$ & $\begin{array}{l}\text { - Na checklist adicionada no cartão que representa um marco, são adicionados } \\
\text { itens com a identificação das tarefas que precisam ser executadas para alcance } \\
\text { daquele marco. }\end{array}$ \\
\hline $\begin{array}{l}\text { Lista de } \\
\text { Riscos }\end{array}$ & Lista & $\begin{array}{l}\text { - Para cada projeto estratégico é criada uma Lista de Riscos. } \\
\text { - No quadro referente ao projeto estratégico, é adicionada uma lista denomina- } \\
\text { da Pontos de Atenção que corresponde a Lista de Riscos. }\end{array}$ \\
\hline Risco & Cartão & $\begin{array}{l}\text { - Na lista denominada Pontos de Atenção, são adicionados cartões que repre- } \\
\text { sentam os riscos do projeto estratégico. }\end{array}$ \\
\hline $\begin{array}{l}\text { Lista de Co- } \\
\text { nhecimento }\end{array}$ & Lista & $\begin{array}{l}\text { - Para cada projeto estratégico é criado uma Lista de Conhecimento. } \\
\text { - No quadro referente ao projeto estratégico, é adicionada uma lista denomina- } \\
\text { da Lições Aprendidas, a qual corresponde a Lista de Conhecimento. }\end{array}$ \\
\hline
\end{tabular}




\begin{tabular}{|l|l|l|}
\hline $\begin{array}{l}\text { Lição Apren- } \\
\text { dida }\end{array}$ & Cartão & $\begin{array}{l}\text { - Na lista denominada Lições Aprendidas, são adicionados cartões que repre- } \\
\text { sentam as lições aprendidas e boas práticas que surgem ao longo do projeto } \\
\text { estratégico. }\end{array}$ \\
\hline $\begin{array}{l}\text { Repositório } \\
\text { de Documen- } \\
\text { tos }\end{array}$ & Lista & $\begin{array}{l}\text { - Para cada projeto estratégico é criado um Repositório de Documentos. } \\
\text { - No quadro referente ao projeto estratégico, é adicionada uma lista denomina- } \\
\text { da Documentos, a qual serve como um Repositório de Documentos do pro- } \\
\text { jeto estratégico. }\end{array}$ \\
\hline Documento & Cartão & $\begin{array}{l}\text { - Na lista denominada Documentos, são adicionados cartões que representam } \\
\text { os documentos que são produzidos ou consumidos no projeto estratégico, tais } \\
\text { como, TAP, TEP, Atas de Reunião, etc. } \\
\text { Em cada cartão que representa um documento, deve ser adicionado um Ane- } \\
\text { xo para upload do arquivo correspondente ou inserção do link (URL) para } \\
\text { uma pasta virtual (ex: Dropbox, Sítio Web) onde se encontra o arquivo. }\end{array}$ \\
\hline
\end{tabular}

Fonte: Autor (2017)

A Figura 4 apresenta um exemplo da representação de elementos do método LeanGov. BR em componentes da ferramenta Trello. No lado esquerdo da Figura 4, um Quadro Kanban do projeto é apresentado através de listas que descrevem a situação de cada marco representado por um cartão. No lado direito da Figura 4, estão as tarefas, representadas por itens de uma checklist (lista de checagem), que são necessárias para o alcance de um marco específico.

Figura 4 - Exemplo de marcos e tarefas
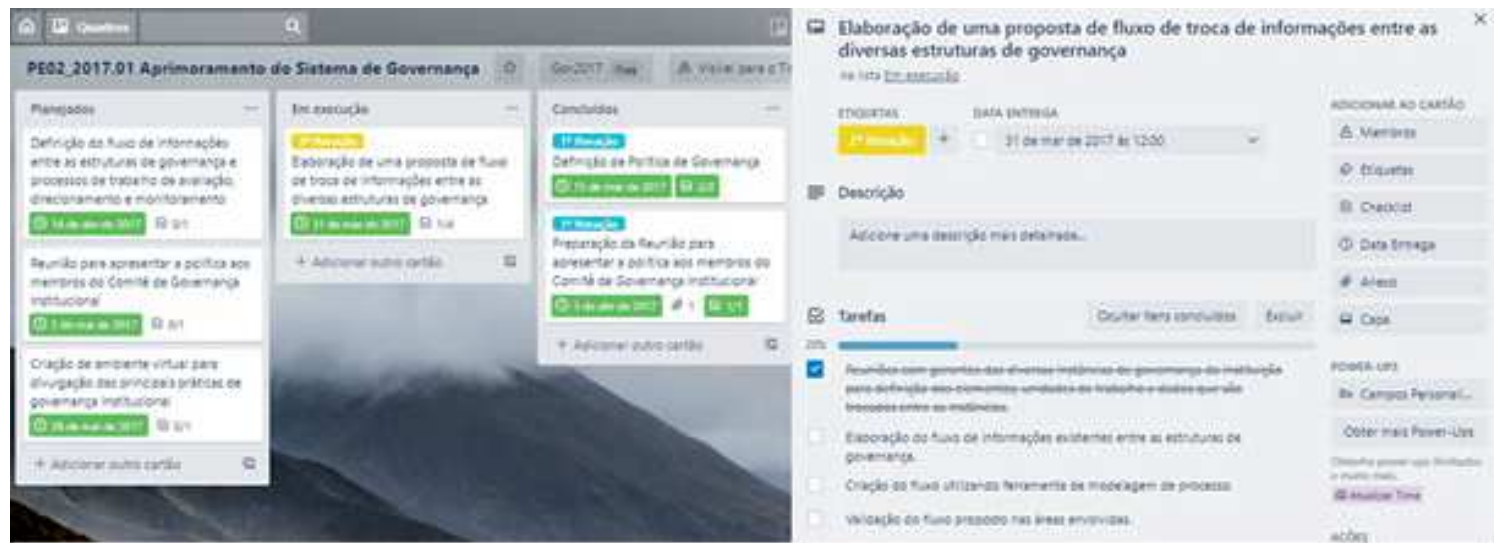

Fonte: Autor (2019)

O suporte tecnológico da ferramenta Trello ao método LeanGov.BR fornece uma maneira de acompanhar o desempenho de um projeto estratégico de cada vez e não é possível monitorar o desempenho do portfólio de projetos estratégicos de um programa em uma única interface de usuário. A tarefa de consolidar informações do portfólio de projetos estratégicos seria demorada e trabalhosa, pois seria necessário acessar manualmente cada quadro que representa um projeto estratégico e analisar o desempenho individual. Para mitigar essa restrição, uma ferramenta de software denominada NumClick foi desenvolvida no contexto deste trabalho, que consiste em um monitor de desempenho do portfólio de projetos de um programa a partir das informações do repositório do Trello. O NumClick apresenta o status de projetos e programas estratégicos por meio de painéis gráficos (dashboards). A Figura 5 mostra exemplos de painéis de monitoramento de desempenho do portfólio de projetos estratégicos associados a um programa, a partir de dados coletados nos cartões do Trello que representam os marcos. 


\section{Aplicação do Método LeanGov.BR}

A aplicação do método LeanGov.BR foi realizada em uma organização pública do poder Judiciário Federal no estado de Pernambuco, perante autorização da alta gestão. O método LeanGov.BR está sendo utilizado para controlar os projetos estratégicos desta organização desde 2017, porém serão apresentados os resultados apenas dos dois primeiros anos de uso.

As etapas que nortearam a aplicação do método LeanGov.BR foram: (1) Diagnóstico da situação atual da governança na organização; (2) Capacitação das equipes envolvidas; (3) Configuração do Trello; (4) Ciclo de planejamento dos programas e projetos estratégicos; e (5) Ciclo de execução dos programas e projetos estratégicos.

Figura 5 - Painel de Desempenho dos Programas Estratégicos
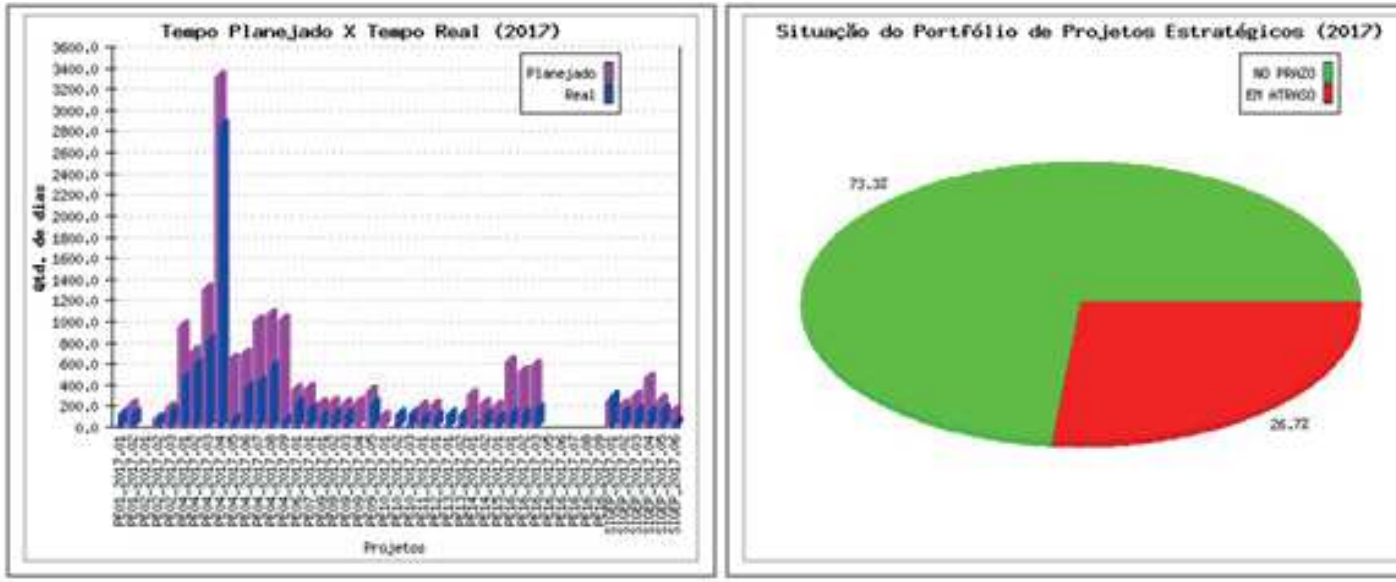

Fonte: Autor (2019)

4.1 Diagnóstico da situação atual da governança na organização

O diagnóstico de governança iniciou com a aplicação de dois questionários direcionados para audiências distintas: (1) alta gestão, composto pela autoridade máxima e dirigentes do primeiro escalão da organização; e (2) gestores dos programas e projetos estratégicos. A Tabela 2 apresenta o quantitativo de respondentes.

Tabela 2 - Número de Respondentes do Questionário de Governança

\begin{tabular}{|c|c|c|c|}
\hline Público-alvo & Total & Respondentes & Adesão \\
\hline Alta Gestão & 05 & 05 & $100 \%$ \\
\hline Gestores de Programas/Projetos & 30 & 16 & $53 \%$ \\
\hline
\end{tabular}

Fonte: Autor (2019)

A partir da análise das respostas dos questionários, algumas deficiências de governança foram identificadas, dentre elas destacam-se: fraco envolvimento da alta gestão no planejamento e no acompanhamento dos projetos estratégicos; dificuldade para desdobrar a estratégia organizacional; dificuldades para planejar projetos com longa duração; impossibilidade dos gestores de projetos em dedicar-se exclusivamente ao projeto devido ao acúmulo das atividades diárias 
com as atividades de gestão dos projetos; cultura burocrática da organização; e necessidade de mudanças culturais para ganho em agilidade. A Figura 6 apresenta o Mapa da Empatia produzido que resume as necessidades, atitudes, ambiente de negócio, ganhos e fraquezas reportados ou percebidos pelos participantes.

Figura 6 - Mapa da empatia dos atores de governança

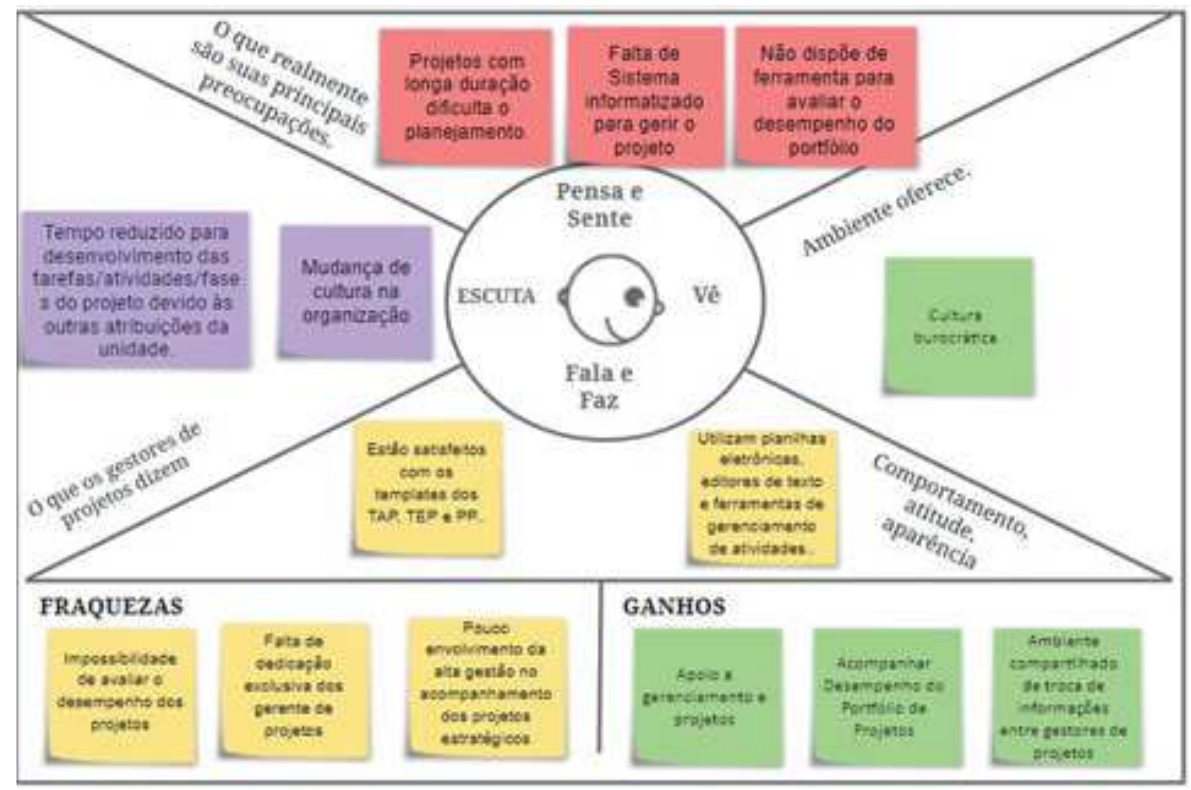

Fonte: Autor (2019)

O diagnóstico prosseguiu com a criação de um grupo focal para atuar na identificação da causa-raiz relacionada ao problema da elevação da taxa de insucesso dos projetos estratégicos. A Figura 7 mostra o Diagrama de Ishikawa resultante da atuação do grupo focal, no qual foram destacadas as principais causas que mais contribuíram para o insucesso de projetos estratégicos. Os resultados obtidos no diagnóstico reforçaram a necessidade de implantação de uma abordagem de governança mais eficiente que mitigasse os problemas identificados.

Figura 7 - Diagrama de Ishikawa

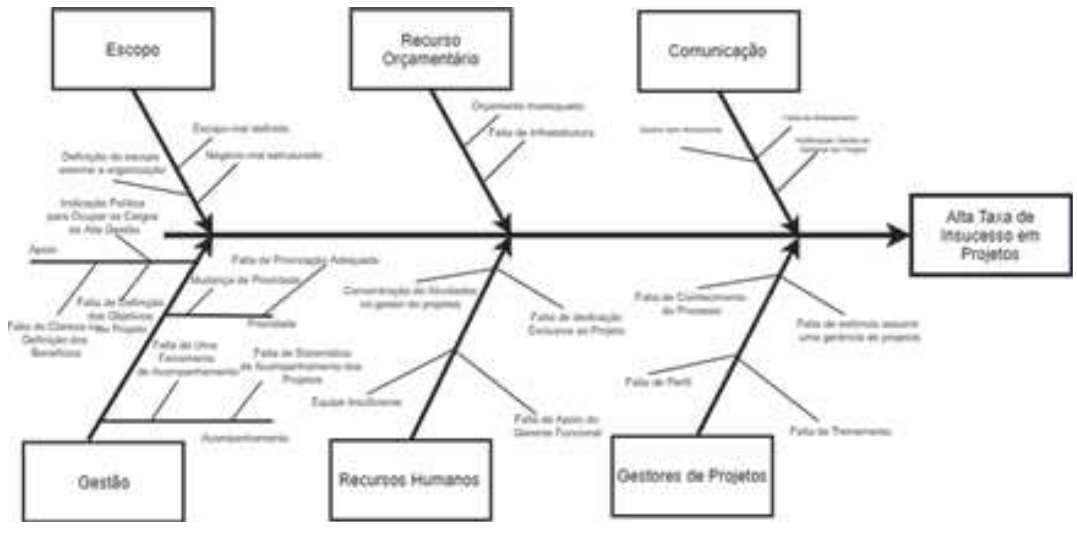

Fonte: Autor (2019) 
4.2 Capacitação das equipes envolvidas

A capacitação teve uma carga horária de 16 horas para um público-alvo de 30 pessoas que desempenham diferentes papéis ao longo da execução do projeto.

\subsection{Configuração do Trello}

A configuração do Trello consistiu em criar as estruturas necessárias para cadastramento e monitoramento dos projetos estratégicos na ferramenta. A seguir são apresentadas as etapas realizadas para configuração do Trello: (1) Criação do Portfólio Estratégico de Projetos; (2) Criação do Kanban para cada Projeto Estratégico; (3) Definição dos Marcos e Tarefas e (4) Criação das listas de gerenciamento de riscos e das lições aprendidas.

4.4 Ciclo de planejamento de programas e projetos estratégicos

O estudo teve um período de duração de aproximadamente 2 anos, tendo iniciado em março/2017 e finalizado em dezembro/2018. A Tabela 3 mostra os ciclos de planejamento dos programas e dos projetos estratégicos.

Tabela 3 - Ciclo de Planejamento dos Programas e Projetos Estratégicos.

\begin{tabular}{|c|c|c|c|c|}
\hline $\begin{array}{c}\text { Ciclo de } \\
\text { planejamento }\end{array}$ & Período & Ano base & $\begin{array}{c}\text { Quantidade de } \\
\text { Programas }\end{array}$ & $\begin{array}{c}\text { Quantidade de } \\
\text { projetos } \\
\text { estratégicos }\end{array}$ \\
\hline $1^{\circ}$ Ciclo & Março/2017 & 2017 & 11 & 30 \\
\hline $2^{\circ}$ Ciclo & Dezembro/2017 & 2018 & 17 & 42 \\
\hline
\end{tabular}

Fonte: Autor (2019)

4.5 Ciclo de execução de programas e projetos estratégicos

Os ciclos de execução, com periodicidade trimestral, consistiram do desenvolvimento incremental do escopo dos projetos estratégicos. A Tabela 4 mostra os períodos de realização do ciclo de execução no ano 2017.

Tabela 4 - Ciclo de Execução dos Programas e Projetos Estratégicos.

\begin{tabular}{|c|c|c|c|}
\hline Ciclo de execução & Início & Fim & $\begin{array}{c}\text { Quantidade de } \\
\text { projetos estratégicos }\end{array}$ \\
\hline $1^{\circ}$ Ciclo & Março/2017 & Maio/2017 & 30 \\
\hline $2^{\circ}$ Ciclo & Junho/2017 & Agosto/2017 & 26 \\
\hline $3^{\circ}$ Ciclo & Setembro/2017 & Novembro/2017 & 25 \\
\hline
\end{tabular}

Fonte: Autor (2019) 
Os ciclos de execução seguiram o workflow do método LeanGov.BR. O planejamento das iterações iniciava com o desdobramento da Visão numa Lista de Marcos ordenada por prioridade de acordo com a estratégia definida pela alta gestão da organização pública. No Trello, os marcos eram registrados como cartões inseridos na lista "Planejados". Os membros do Time então selecionavam os marcos a serem alcançados na iteração com prazo trimestral. No Trello, os Marcos Selecionados para a iteração eram identificados através de uma etiqueta de cor azul associada aos respectivos cartões que os representavam. Em seguida, os membros do Time juntamente com o Gerente do Projeto Estratégico elaboravam a Lista de Riscos do projeto. No Trello, os riscos eram representados por cartões inseridos na lista denominada "Pontos de Atenção". No Trello, para cada risco eram definidas as ações de tratamento (mitigar, aceitar, transferir e evitar) registradas como itens da checklist do respectivo cartão. No encerramento do planejamento da iteração, os membros do Time definiam as tarefas necessárias para o alcance dos marcos selecionados e então distribuíam as responsabilidades. No Trello, as tarefas eram registradas como itens da checklist do cartão que representava o marco.

A execução das iterações iniciava com os deslocamentos dos marcos contidos na lista "Planejados" para a lista "Em Execução" e com a realização das primeiras tarefas dos marcos em execução pelos devidos responsáveis. No Trello, à medida que as tarefas eram concluídas, o item de checklist correspondente era sinalizado (checked), no cartão que representava o marco. As reuniões de monitoramento das iterações eram apoiadas pela visualização dos quadros Kanban com o status dos marcos e tarefas contidos nos cartões do Trello e pelos dashboards do NumClick. O encerramento das iterações consistia na homologação da entrega pelo Dono do Programa e com verificação em retrospectiva do que funcionou e aspectos a melhorar. A Figura 8 mostra o Quadro Kanban do projeto "PE02_2017.01_Estruturação do Sistema de Governança Institucional” ao final do primeiro ciclo de execução.

Figura 7 - Quadro do Projeto ao final do primeiro ciclo de execução.

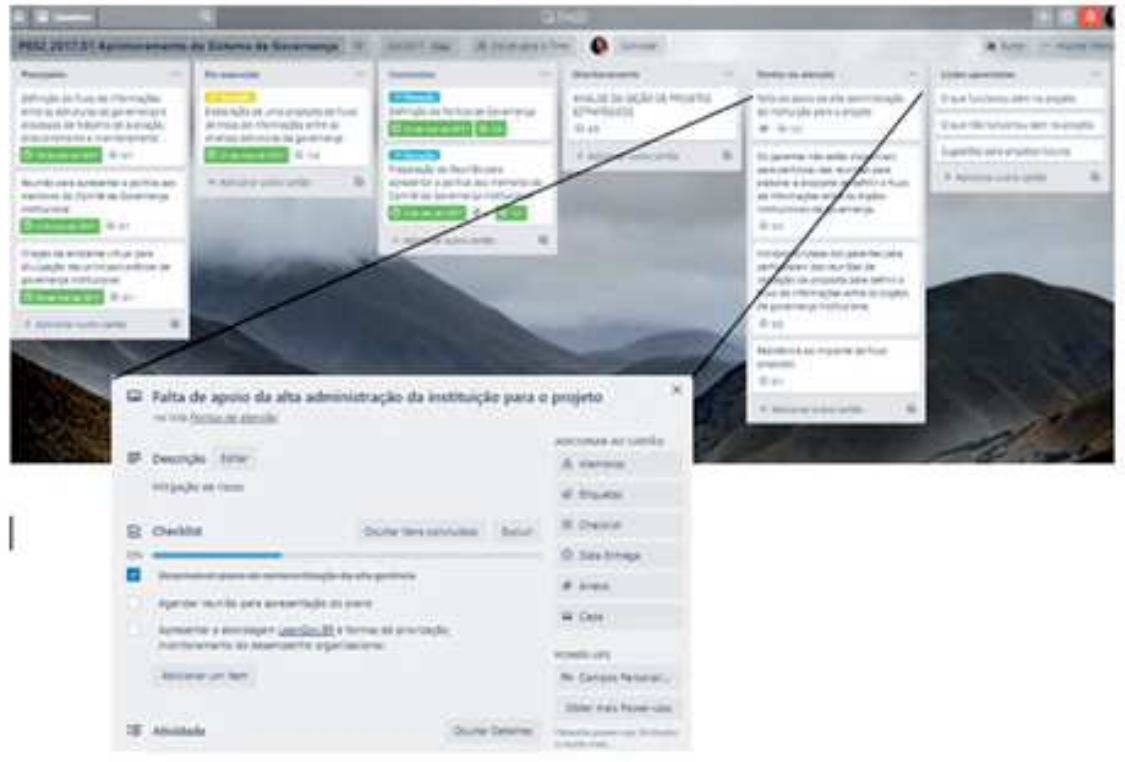

Fonte: Autor (2019) 


\section{Resultados}

A avaliação dos resultados obtidos com a aplicação do método LeanGov.BR ocorreu em duas etapas: (1) Obtenção e análise de feedback dos participantes; e (2) Análise quantitativa do desempenho dos programas e projetos estratégicos.

\subsection{Obtenção e análise de feedback dos participantes}

O feedback foi obtido durante as reuniões mensais de monitoramento dos projetos. Essas reuniões contaram com a presença de Donos de Programa, Gerente de Projetos Estratégicos e por representante do Escritório de Projetos. Este último foi responsável por realizar as entrevistas, coletar o feedback, e registrar e categorizar os resultados. As principais melhorias introduzidas pelo uso do método LeanGov.BR na organização, citadas pelos participantes, foram:

- Permitiu uma atuação mais rápida na remoção de impedimentos, planejamento mais realista, disseminação das informações e notificação dos envolvidos.

- Permitiu um acompanhamento sistemático, organizado e em momento oportuno da situação do projeto, provendo meios de intervenção imediata.

- Contribuiu para armazenar num único ambiente as evidências do projeto, os riscos e o tratamento facilitando a justificativa das ações adotadas pelo gerente de projetos ao longo da condução do projeto.

- Permitiu o monitoramento do desempenho do portfólio de projetos e ampliou a percepção de contribuição para alcance dos objetivos estratégicos da organização.

A partir do feedback obtido e da experiência de uso do LeanGov.BR durante os ciclos de planejamento e execução dos programas e projetos estratégicos, foi possível inferir que a abordagem contribuiu para atenuar causas de problemas identificados no diagnóstico de governança da organização pública, entre as quais: Notificação tardia dos gerentes de projeto; Falta de uma sistemática de acompanhamento dos projetos; Falta de priorização adequada; Falta de definição dos objetivos do projeto; Falta de clareza na definição dos benefícios do projeto; Falta de conhecimento do processo; Gestor sem autonomia; Falta de alinhamento entre os gerentes e o time no kickoff do projeto; e Falta de ferramenta de acompanhamento de projetos.

\subsection{Análise quantitativa do desempenho de programas e projetos estratégicos}

No primeiro ano (2017) de uso do método LeanGov.BR, a taxa de conclusão de projetos foi de $55 \%$ e a taxa de cancelamentos de projetos foi de $25 \%$. Esses índices provavelmente estavam relacionados a falhas no planejamento dos projetos e a imaturidade organizacional em adotar paradigma ágil e enxuto (lean). No segundo ano (2018) de adoção do método LeanGov. BR na organização pública, a taxa de conclusão de projetos aumentou para $88 \%$ e não houve projetos cancelados. A decomposição de projetos complexos em projetos com menor escopo e planejamento de entregas mais frequentes em períodos mais curtos, contribuíram para a melho- 
ria o desempenho de programas e projetos estratégicos.

O Gráfico 1 demonstra a evolução do desempenho do portfólio de projetos estratégicos no período compreendido entre 2017 e 2018. A análise do gráfico permite inferir que o LeanGov.BR teve papel fundamental e contribuiu para elevação dos percentuais de conclusão dos projetos, redução das falhas no planejamento dos projetos e diminuição no número de cancelamentos. $\mathrm{O}$ experimento permitiu deduzir que os princípios, práticas e ferramentas difundidas pelas metodologias ágeis e Lean provavelmente podem ter sua aplicabilidade ampliada para outras áreas da organização, não sendo restritas para a Área de Tecnologia da Informação (TI) ou ao meio industrial. O LeanGov.BR demonstra-se uma abordagem flexível e adaptável ao contexto institucional. $\mathrm{O}$ experimento demonstrou que a abordagem mostra-se satisfatória quando aplicada a projetos corporativos com temática diversificada.

Gráfico 1 - Evolução do Desempenho dos Projetos Estratégicos.

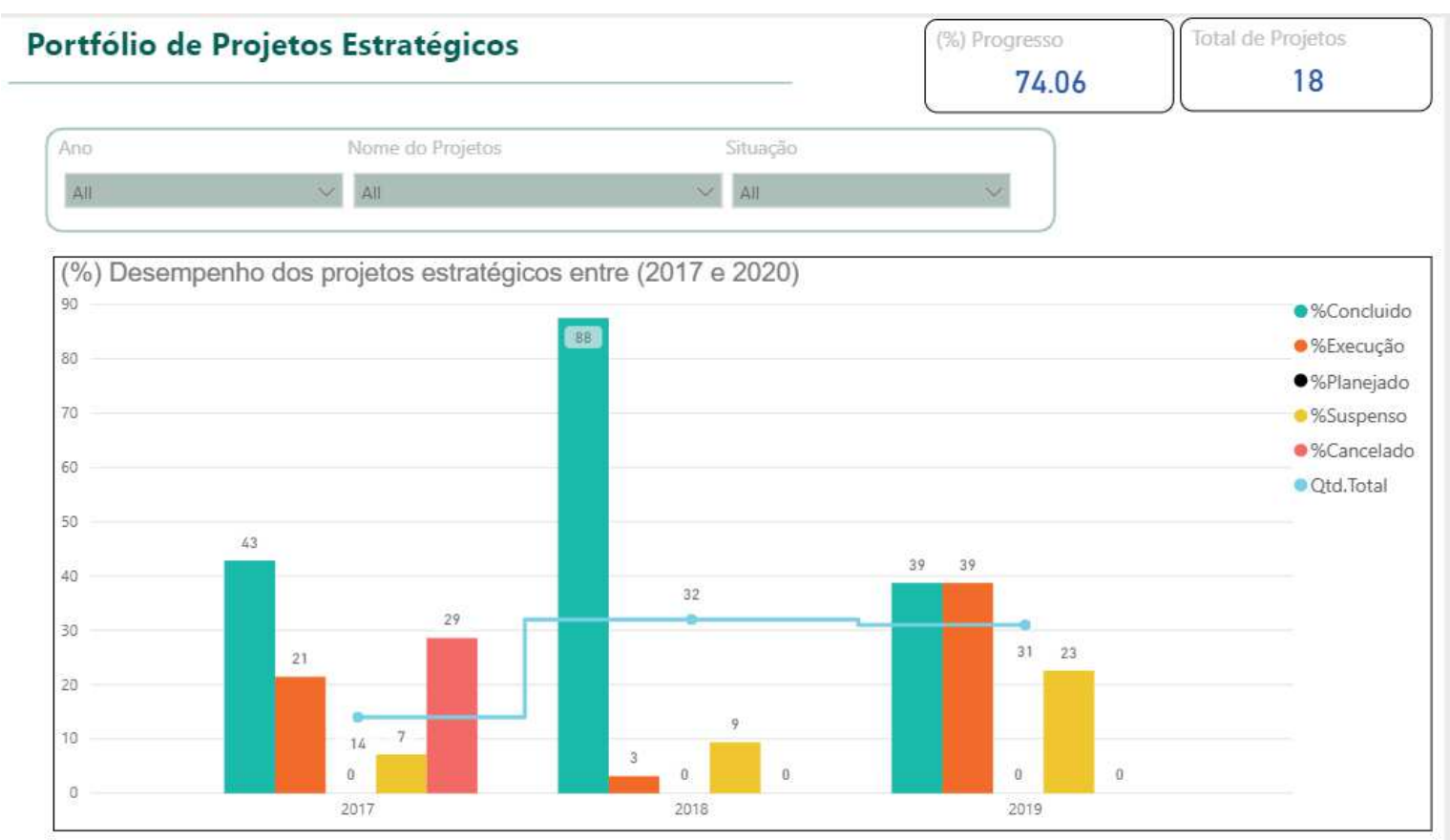

Fonte: Autor (2020)

Os resultados e discussões acima permitem inferir que a abordagem LeanGov.BR teve um papel fundamental para elevação dos índices de conclusão de projetos estratégicos e para redução dos índices de cancelamento de projetos estratégicos. A instituição avaliada alinha cada um dos objetivos estratégicos do PEI aos programas e, em seguida, desdobra o programa em projetos estratégicos e em iniciativas que juntos contribuem para o alcance dos próprios objetivos estratégicos. A elevação nos índices de projetos estratégicos concluídos (de 56,67\% em 2017 para 88,10\% em 2018) permitiu deduzir que o LeanGov.BR influenciou positivamente, contribuindo consequentemente para o alcance da estratégia organizacional.

Os resultados obtidos conferem que à forma de trabalho mais participativa, na qual as responsabilidades são compartilhadas entre as partes envolvidas influenciou positivamente o desempenho dos projetos estratégicos. Verificou-se também que o planejamento evolutivo funcionou de maneira mais aderente à realidade organizacional. Um fator que colaborou para o bom funcionamento desta forma de trabalho foi a priorização trimestral das entregas que 
refletiu na diminuição das falhas de planejamento do escopo e na redução dos índices de cancelamento de projetos. Além disso, o componente tecnológico da abordagem também proporcionou maior visibilidade das responsabilidades de cada um dos integrantes da equipe, gerando maior comprometimento e forneceu novas formas de acompanhamento dos projetos. Uma vez que todos os envolvidos, desde a equipe de projeto, gerente de projetos, gestores de programas estratégicos até a parte cliente, podem acompanhar o desempenho e a situação das entregas, os riscos e as intercorrências. Ademais, possibilitou a rastreabilidade de toda a comunicação trocada entre as partes interessadas, gerando evidências para possíveis auditorias dos órgãos de controle interno e externo. A conclusão geral a que se chegou com o experimentação do método foi que o LeanGov.BR possui grande potencial para ser utilizado como instrumento de apoio a governança pública, já que facilita o fluxo de trocas de informações entre as diversas instâncias de governança, permite um ágil monitoramento dos riscos estratégicos, permite uma análise de alcance dos objetivos estratégicos da instituição a partir da perspectiva de desempenho dos projetos estratégicos.

\section{Conclusão}

O método LeanGov.BR se propõe a resolver os problemas encontrados nas abordagens de governança tradicionais, tais como, complexidade, burocracia, processos complexos, excesso de controles e necessidade de altos investimentos, por meio de uma abordagem enxuta para controle da estratégia da governança pública brasileira. Para tanto, o método LeanGov.BR está alinhado ao modelo de referência para governança pública (TCU, 2014).

As principais contribuições deste trabalho são: especificação do método LeanGov.BR através de elementos estruturais, comportamentais e tecnológicos, baseados na agilidade e no pensamento enxuto; execução e avaliação do método proposto em uma organização pública do poder judiciário federal brasileiro, na qual foi possível obter feedback positivo em relação a melhorias do desempenho organizacional provocadas pelo uso do LeanGov.BR; configuração da ferramenta de software para gestão ágil de atividades Trello para dar suporte tecnológico ao método LeanGov.BR; e desenvolvimento da ferramenta de software denominada NumClick que provê à alta gestão painéis e relatórios com o desempenho da estratégia organizacional a partir dos dados do Trello.

Entre as limitações do trabalho estão a implantação e avaliação do método LeanGov. BR em apenas uma organização pública; e suporte apenas ao mecanismo de estratégia do referencial de governança pública do TCU. As oportunidades de trabalhos futuros relacionadas às limitações são: experimentar o método LeanGov.BR em outras organizações públicas de modo a avaliar com maior precisão a efetividade da abordagem por meio da análise dos resultados qualitativos e quantitativos obtidos; estender a abordagem para dar suporte aos demais mecanismos (liderança e controle) do referencial de governança pública; estender abordagem para contemplar o planejamento orçamentário; avaliar a influência do componente cultura organizacional no uso do método LeanGov.BR; e analisar o impacto do uso do método LeanGov.BR no levantamento de governança pública do TCU. 


\section{Referências}

Beck, K. (1999). Programação Extrema Explicada. Bookman.

BSI. (1997). Balanced ScoreCard Institute. Acesso em Janeiro de 2018. Disponível em http:// www.balancedscorecard.org/BSC-Basics/About-the-Balanced-Scorecard.

Chiavenato, I; Sapiro, A. (2003). Planejamento estratégico. Rio de Janeiro: Elsevier.

CIPFA. (2004). Chartered Institute of Public Finance and Accountancy. The good governance standard for public services. Acesso em março de 2018. Disponivel em: http://www.jrf.org.uk/ system/files/1898531862.pdf

Cockburn, A. (2000). A Agile Software Development Draft version: 3b. Highsmith Series. Acesso em Dezembro de 2017. Disponível em http://zsiie.icis.pcz.pl/ksiazki/Agile\%20Software\%20 Development.pdf

Constituição Federal do Brasil. (1988). LOA - Lei de Diretrizes Orçamentárias. Acesso em Fevereiro de 2018. Disponível em http://www.senado.leg.br/

COSO.(2004). Committee of Sponsoring Organizations of the Treadway Commission. Enterprise risk management: integrated framework. Acesso em março de 2018. Disponível em: http://www.coso.org/guidance.htm.

COSO. (2013). Committee of Sponsoring Organizations of the Treadway Commission. Internal control: integrated framework. Acesso em março de 2018. Disponível em: http://www.coso. org/ guidance.htm.

IFAC. (1977). International Federation of Accountants. Acesso em Janeiro de 2018. Disponível em https://www.ifac.org/

Lean Enterprise Institute. (1998). Lean Enterprise Institute . Acesso em Janeiro de 2018, disponível em https://www.lean.org

Luna, A. (2009). MAnGve: Um Modelo para Governança Ágil em Tecnologia da Informação e Comunicação. Dissertação: Mestrado em Ciência da Computação. Recife, PE, Brasil.

Luna, A. J., Kruchten, P., Pedrosa, M. L., Neto, H. R., \& Moura, H. P. (2014). State of the Art of Agile Governance: A Systematic Review. International Journal of Computer Science and Information Technology, v.6, n.5, p. 121-141.

Manifesto Ágil. (2001). Manifesto para o desenvolvimento ágil de software. Acesso em Dezembro de 2017, disponível em www.manifestoagil.com.br

MICHAELIS. (2018). Moderno Dicionário da Língua Portuguesa. Acesso em: 10 de agosto de 
2018. Disponível em: http://michaelis.uol.com.br/moderno/portugues/index.php.

OECD. (2015). Principles of Corporate Governance. Acesso em Fevereiro de 2018, disponível em, http://www.oecd.org/corporate/principles-corporate-governance.htm.

OGC. (2009). Prince2: A Pocket Guide. Acesso em Janeiro de 2018, disponível em http://www. vanharen.net/Samplefiles/9789087535445 prince2-edition-2009-a-pocket-guide.pdf

OMG. (2008). SPEM 2.0 - Software \& Systems Process Engineering Metamodel. Disponível em http://www.omg.org/spec/SPEM/About-SPEM/

Pedrão, L. C. (2014). Gerenciamento de Projetos lean utilização otimizada de recursos garante sucesso na gestão de projetos. Pós-MBA em Gerenciamento Avançado de Projetos, na instituição IBE FGV. Campinas/SP.

PMI. (2013). Um Guia do Conjunto de Conhecimentos em Gerenciamento de Projetos (Guia PMBOK®) (5th ed.). Standard: PMI.

Putnik, G. D., \& Putnik, Z. (2012). Lean vs agile in the context of complexity management in organizations. The Learning Organization, 19(3), 248-266. https://doi. org/10.1108/09696471211220046

Schwaber, K. (1995). Scrum. Acesso em Janeiro de 2018, disponível em https://www.scrum.org/

Shingo, S. (2007). Sistema Toyota de Produção: o ponto de vista da engenharia de produção. Porto Alegre: Bookman.

TCU. (1983). Tribunal de Contas da União. Acesso em Dezembro de 2017, disponível em http:// portal.tcu.gov.br/

TCU. (2014). Referencial básico de governança aplicável a órgãos e entidades da administração pública / Tribunal de Contas da União. Versão 2. Brasília, Brasil.

TCU. (2017). Tribunal de Contas da União: acórdão. Disponível em: http://sintse.tse.jus.br/ documentos/2017/Mai/12/acordao-no-882-2017-plenario-levantamento-de-auditoria-diversasunidades-jurisdicionadas-em-todos-os-poderes-tre-pa

The Standish Group. (1995). Chaos Report. Acesso em julho de 2018. Disponível em https:// www.standishgroup.com/.

Trello. (2011). Trello Software Tool. Acesso em Fevereiro de 2018. Disponível em https://trello. $\underline{\mathrm{com} / .}$

Wodtke, C. (2016). Introduction to OKR. Sebastopol/CA. 
